\title{
STABILITY FOR THE PERIODIC CAMASSA-HOLM EQUATION
}

\author{
JONATAN LENELLS
}

\begin{abstract}
We use integrability to prove the stability of smooth periodic solutions of the Camassa-Holm equation. In particular, the smooth periodic traveling wave solutions are shown to be orbitally stable.
\end{abstract}

\section{Introduction}

The Camassa-Holm equation

$$
u_{t}-u_{t x x}+3 u u_{x}=2 u_{x} u_{x x}+u u_{x x x}, \quad x \in \mathrm{R}, \quad t>0,
$$

is a model for the propagation of shallow water waves over a flat bottom, $u(x, t)$ representing the water's free surface in non-dimensional variables [2]. Equation (1.1) was first obtained [19] as an abstract bi-Hamiltonian equation with infinitely many conservation laws. Equation (1.1) arises also as a model for nonlinear waves in cylindrical axially symmetric hyperelastic rods, with $u(x, t)$ representing the radial stretch relative to a prestressed state [17]. Moreover, (1.1) is a re-expression of the geodesic flow in the group of compressible diffeomorphisms of the circle [32]. This geometric interpretation leads to a proof that equation (1.1) satisfies the Least Action Principle [10]: a state of the system is transformed to another nearby state through a uniquely determined flow that minimizes the energy (see also [11]). Let us also point out that for a large class of initial data, equation (1.1) is an infinite-dimensional completely integrable Hamiltonian system: by means of an isospectral problem one can convert the equation into a finite or infinite sequence of linear ordinary differential equations which can be trivially integrated (see [6], [12]). Equation (1.1) admits, in addition to smooth waves, a multitude of traveling wave solutions with singularities - peakons, cuspons, stumpons, and composite waves [2], [24]. The Camassa-Holm equation models wave breaking [2], [5], [7], [8], [9],

Received August 15, 2004. 
[18], [27], [30] and admits wave solutions that exist indefinitely in time [5], [8]. Let us also point out that associated to (1.1) there is a whole hierarchy of integrable equations [20].

We will consider stability of smooth periodic solutions of the CamassaHolm equation. In [16] it was shown that the smooth solitary waves of (1.1) are orbitally stable. Moreover, the peaked solutions, whether solitary waves or periodic waves, are also orbitally stable [14], [15], [21], [22]. The stability result presented in this paper applies to all (traveling waves or not) smooth periodic solutions with positive momentum $m=u-u_{x x}>0$.

In Sections 2 and 3 we review notation and some aspects of the isospectral problem associated to (1.1). Using the recently established link between Camassa-Holm and KdV [26], we derive in Section 4 a useful expression for the eigenvalue asymptotics of the isospectral problem. Section 5 shows that for a large class of initial data equation (1.1) can be viewed as a motion on infinite-dimensional tori. Since a solution of (1.1) remains on the same torus for all times, stability can geometrically be expressed by saying: If two different initial data are close, then so are the tori on which they lie. Our main result is stated in Section 6: the Camassa-Holm equation is stable in this sense. As a direct consequence it follows that the periodic smooth traveling wave solutions of (1.1) are orbitally stable. The proof, based on inverse spectral theory, is presented in Section 7.

Our approach is inspired by [29], where a similar proof of stability was outlined for the $\mathrm{KdV}$ equation.

\section{Preliminaries}

We let $\mathrm{S}$ be the circle of length 1 . The space $C^{k}(\mathrm{~S}), k \geq 0$, consists of all $k$ times continuously differentiable functions $f$ with

$$
\|f\|_{C^{k}(\mathbf{S})}=\sum_{j=0}^{k} \max _{x \in \mathbf{S}}\left|f^{(j)}(x)\right|<\infty .
$$

$C^{\infty}(\mathrm{S})$ denotes the set of smooth functions on $\mathrm{S}$. Let $\mathscr{D}^{\prime}(\mathrm{S})$ be the space of distributions on $\mathrm{S}$, i.e. continuous linear functionals on $C^{\infty}(\mathrm{S})$. We denote, for $s \in \mathrm{R}$, by $H^{s}(\mathrm{~S})$ the space of $f \in \mathscr{D}^{\prime}(\mathrm{S})$ such that

$$
\|f\|_{H^{s}}^{2}=\sum_{k=-\infty}^{\infty}\left(1+(2 \pi k)^{2}\right)^{s}|\hat{f}(k)|^{2}<\infty,
$$

where the Fourier coefficients are defined by

$$
\hat{f}(k)=\langle f, \exp (-2 \pi i k \cdot)\rangle_{L^{2}(\mathbf{S})}, \quad k \in \mathbf{Z} .
$$




\section{Isospectral Problem}

In terms of the momentum $m=u-u_{x x}$, equation (1.1) can be expressed (cf. [3]) as the condition of compatibility between

$$
\psi_{x x}=\frac{1}{4} \psi+\lambda m \psi
$$

and

$$
\psi_{t}=\left(\frac{1}{2 \lambda}-u\right) \psi_{x}+\frac{1}{2} u_{x} \psi
$$

More precisely, if $\psi(x, t)$ solves (3.1) and (3.2), then $\left(\psi_{x x}\right)_{t}=\left(\psi_{t}\right)_{x x}$ if and only if $u$ is a solution to the Camassa-Holm equation.

The isospectral problem (3.1) has two normalized solutions $\psi_{1}(x, \lambda)$ and $\psi_{2}(x, \lambda)$ determined by the conditions $\psi_{1}(0, \lambda)=1, \psi_{1 x}(0, \lambda)=0$; $\psi_{2}(0, \lambda)=0, \psi_{2 x}(0, \lambda)=1$. The periodic spectrum consists of periodic eigenvalues (numbers $\lambda$ for which there is a periodic eigenfunction: $\psi(0)=$ $\psi(1)$ and $\left.\psi_{x}(0)=\psi_{x}(1)\right)$ and antiperiodic eigenvalues (numbers $\lambda$ for which there is an antiperiodic eigenfunction: $\psi(0)=-\psi(1)$ and $\left.\psi_{x}(0)=-\psi_{x}(1)\right)$. The Dirichlet spectrum is determined by solving (3.1) with the boundary conditions $\psi(0)=\psi(1)=0$; it comprises the roots of $\psi_{2}(1, \lambda)=0$. The discriminant of (3.1) is

$$
\Delta(\lambda)=\frac{1}{2}\left[\psi_{1}(1, \lambda)+\psi_{2 x}(1, \lambda)\right], \quad \lambda \in \mathrm{C} .
$$

Floquet's theorem implies that the periodic (antiperiodic) eigenvalues of (3.1) are exactly the zeros of $\Delta(\lambda)=1(\Delta(\lambda)=-1)[28]$.

The qualitative structure of the spectrum of (3.1) was described in [4]: If $m \geq 0$ there is a simple periodic ground state $\lambda_{0}$ followed by alternately anti-periodic and periodic pairs

$$
\cdots<\lambda_{4} \leq \lambda_{3}<\lambda_{2} \leq \lambda_{1}<\lambda_{0}<0,
$$

of simple or double eigenvalues accumulating at $-\infty$. There is precisely one simple eigenvalue $\mu_{n}$ of the Dirichlet spectrum in each interval $\left[\lambda_{2 n}, \lambda_{2 n-1}\right]$, $n=1,2, \ldots$, and no others. If $m \leq 0$ the pattern is simply reflected in $\lambda=0$.

Remark 1 . In the sequel we study only the case of smooth positive momentum $0<m=u-u_{x x} \in C^{\infty}$ (S). If the smooth initial data for equation (1.1) satisfies $m_{0}=u_{0}-u_{0 x x}>0$, then $m$ remains strictly positive and smooth for all times [9]. 
The functions $\Delta(\lambda), \psi_{2}(1, \lambda)$, and $\psi_{1 x}(1, \lambda)$ are entire analytic function of order $\frac{1}{2}$ of the complex variable $\lambda$ [4]. Therefore, since the roots of $\Delta^{2}(\lambda)-1=$ 0 are the $\lambda$ 's, the Weierstrass factorization theorem yields

$$
\Delta^{2}(\lambda)-1=\sinh ^{2}(1 / 2)\left(1-\frac{\lambda}{\lambda_{0}}\right) \prod_{n \geq 1}\left(1-\frac{\lambda}{\lambda_{2 n-1}}\right)\left(1-\frac{\lambda}{\lambda_{2 n}}\right)
$$

Similarly, since $\psi_{2}(1,0)=2 \sinh (1 / 2)$ and the roots of $\psi_{2}(1, \lambda)=0$ are the eigenvalues $\left\{\mu_{n}\right\}_{n=1}^{\infty}$ of the Dirichlet spectrum, we get

$$
\psi_{2}(1, \lambda)=2 \sinh (1 / 2) \prod_{n \geq 1}\left(1-\frac{\lambda}{\mu_{n}}\right) .
$$

\section{Eigenvalue Asymptotics}

The bi-Hamiltonian structure of (1.1) gives rise to an infinite sequence of quantities

$\ldots, H_{-2}[m], H_{-1}[m], H_{0}[m], H_{1}[m], H_{2}[m], H_{3}[m], \ldots$,

conserved under the flow of (1.1) (see [2], [25]). The first few conservation laws in this sequence are

$$
\begin{gathered}
H_{-2}[m]=-\frac{1}{16} \int_{\mathrm{S}}\left(\frac{4}{\sqrt{m}}+\frac{m_{x}^{2}}{16 m^{5 / 2}}\right) d x, \quad H_{-1}[m]=\int_{\mathrm{S}} \sqrt{m} d x, \\
H_{0}[m]=\int_{\mathrm{S}} m d x, \quad H_{1}[m]=\frac{1}{2} \int_{\mathrm{S}} m u d x, \quad H_{2}[m]=\frac{1}{2} \int_{\mathrm{S}}\left(u^{3}+u u_{x}^{2}\right) d x .
\end{gathered}
$$

The Liouville transformation

$$
y=\int_{0}^{x} \sqrt{m(\xi)} d \xi, \quad \phi(y)=m(x)^{1 / 4} \psi(x),
$$

converts the isospectral problem (3.1) for the Camassa-Holm equation into the isospectral problem for the $\mathrm{KdV}$ equation

$$
-\phi_{y y}+Q(y) \phi=v \phi,
$$

where $v=-\lambda$ and

$$
Q(y)=\frac{1}{m(x)}\left(\frac{1}{4}-\frac{\left(m(x)^{-1 / 4}\right)_{x x}}{m(x)^{-1 / 4}}\right) .
$$


Equation (4.2) is a Hill's equation on an interval of length $L=H_{-1}[\mathrm{~m}]=$ $\int_{\mathrm{S}} \sqrt{m(\xi)} d \xi$. Hence the periodic and antiperiodic eigenvalues of (4.2) have the common expansion (see [31])

$$
\begin{aligned}
v_{2 n}, v_{2 n-1}=\frac{n^{2} \pi^{2}}{L^{2}}+\frac{\bar{H}_{0}}{L}+\left(L \bar{H}_{1}-\right. & \left.\frac{1}{2} \bar{H}_{0}^{2}\right)(n \pi)^{-2} \\
& +P\left(\bar{H}_{2}, \bar{H}_{1}, \bar{H}_{0}\right)(n \pi)^{-4}+\cdots,
\end{aligned}
$$

with coefficients expressed in terms of the conservation laws for the $\mathrm{KdV}$ equation

$$
\begin{aligned}
\bar{H}_{0}[Q]=\int_{0}^{L} Q d y, \quad \bar{H}_{1}[Q]= & \frac{1}{2} \int_{0}^{L} Q^{2} d y, \\
& \bar{H}_{2}[Q]=\frac{1}{2} \int_{0}^{L}\left(Q^{3}+\frac{1}{2} Q_{y}^{2}\right) d y, \quad \ldots,
\end{aligned}
$$

and the coefficient of $(n \pi)^{-2 j}$ being a polynomial in $\bar{H}_{0}, \ldots, \bar{H}_{j}$.

Since it was proved in [26] that

$$
\bar{H}_{0}[Q]=-H_{-2}[m], \quad \bar{H}_{1}[Q]=-H_{-3}[m], \quad \bar{H}_{2}[Q]=-H_{-4}[m], \quad \ldots,
$$

we obtain the asymptotic formula

$$
\begin{aligned}
\lambda_{2 n}, \lambda_{2 n-1}=-\frac{n^{2} \pi^{2}}{H_{-1}^{2}}+\frac{H_{-2}}{H_{-1}} & +\left(H_{-1} H_{-3}+\frac{1}{2} H_{-2}^{2}\right)(n \pi)^{-2} \\
& +P\left(H_{-1}, H_{-2}, H_{-3}, H_{-4}\right)(n \pi)^{-4}+\cdots,
\end{aligned}
$$

for the eigenvalues of (3.1). Notice that the coefficient of $(n \pi)^{-2 j}$ depends only on $H_{-1}, \ldots, H_{-j-2}$.

\section{Tori}

The integrability of the Camassa-Holm equation provides a way to view its flow geometrically as a motion on infinite-dimensional tori. Indeed, for a momentarily fixed $m$ let

$$
\ldots<\lambda_{4} \leq \lambda_{3}<\lambda_{2} \leq \lambda_{1}<\lambda_{0}<0,
$$

be the periodic spectrum of the isospectral problem (3.1). Then the manifold $M$ of all potentials giving rise to the same sequence of $\lambda$ 's is a torus $M \subset C^{\infty}(\mathrm{S})$ of dimension $d \leq \infty ; 2 d+1$ being the number of simple periodic eigenvalues. Since the periodic spectrum of (3.1) is preserved under the Camassa-Holm flow a solution of (1.1) remains on the same torus for all times. 
The demonstration that $M$ is a torus proceeds as follows. We introduce the norming constants

$$
c_{n}=\frac{1}{\int_{\mathrm{S}} m(x) \psi_{2}^{2}\left(x, \mu_{n}\right) d x}, \quad n \geq 1 .
$$

If the periodic spectrum and the Dirichlet eigenvalues are known, then $\Delta(\lambda)$ and $\psi_{2}(1, \lambda)$ can be recovered from (3.3) respectively (3.4). But then, as (see [12])

$$
c_{n}=\frac{1}{\psi_{2 x}\left(1, \mu_{n}\right) \dot{\psi}_{2}\left(1, \mu_{n}\right)}=\frac{1}{\left(\Delta\left(\mu_{n}\right) \pm \sqrt{\Delta^{2}\left(\mu_{n}\right)-1}\right) \dot{\psi}_{2}\left(1, \mu_{n}\right)},
$$

also the norming constants are determined up to the sign of $\sqrt{\Delta^{2}\left(\mu_{n}\right)-1}$. The signature of the radical is ambiguous only if $\Delta^{2}\left(\mu_{n}\right) \neq 1$, i.e. only if $\lambda_{2 n}<$ $\mu_{n}<\lambda_{2 n-1}$. This suggests that we open up the closed interval $\left[\lambda_{2 n}, \lambda_{2 n-1}\right]$ into a circle, and place $\mu_{n}$ on the upper or lower semicircle according to the positive or negative determination of the radical (cf. [29]). If $\lambda_{2 n}=\lambda_{2 n-1}$ the circle collapses to a point and may then be ignored.

To show that the map

$$
m \mapsto\left\{\mu_{n}, \sqrt{\Delta^{2}\left(\mu_{n}\right)-1}\right\}_{n=1}^{\infty},
$$

from $M$ into the product of the $d \leq \infty$ nontrivial circles is one-to-one we use the following crucial result.

Lemma 1 ([6]). There is a unique periodic solution $\mu_{n}(t), n \geq 1$, of the system

$$
\frac{d \mu_{n}}{d t}(t)=\frac{\mu_{n}(t)}{\sinh (1 / 2)} \frac{\sqrt{\Delta^{2}\left(\mu_{n}(t)\right)-1}}{\prod_{1 \leq m \neq n}\left(1-\frac{\mu_{n}(t)}{\mu_{m}(t)}\right)} .
$$

which does not pause at simple periodic eigenvalues (i.e. $\frac{d \mu_{n}}{d t}$ has a discrete set of simple roots unless $\left.\lambda_{2 n}=\lambda_{2 n-1}\right)$, whose initial values are $\mu_{n}(0)$ and for which the initial velocities are prescribed by choosing the signature of the radical $\sqrt{\Delta^{2}\left(\mu_{n}\right)-1}$ such that

$$
\sqrt{\Delta^{2}\left(\mu_{n}\right)-1}=\frac{1}{c_{n} \dot{\psi}_{2}\left(1, \mu_{n}\right)}-\Delta\left(\mu_{n}\right)
$$

Moreover,

$$
m(x)=\frac{1}{2} \sum_{n \geq 1}\left[\frac{1}{\mu_{n}(x)}-\left(\frac{1}{\mu_{n}(x)}\right)_{x x}\right]-\frac{1}{4} \sum_{n \geq 0} \frac{1}{\lambda_{n}} .
$$


The point here is that $m$ can be recovered from the norming constants and the periodic and Dirichlet spectra, because $\mu_{n}(t)$ is constructed from just this information: Any point $\left\{\mu_{n}, \sqrt{\Delta^{2}\left(\mu_{n}\right)-1}\right\}_{n=1}^{\infty}$ can be used as initial condition, and once $\mu_{n}(t)$ is obtained from the differential equations (5.3), then $m$ is found by means of the trace formula (5.4). This shows that the map (5.2) is bijective. Hence $M$ can be identified with a torus.

The differential equations (5.3) describe the motion of the Dirichlet eigenvalues under the translation flow $m(x) \mapsto m(x+t)$. Moreover, the eigenvalue $\mu_{n}(t)$ hits both endpoints of the interval $\left[\lambda_{2 n}, \lambda_{2 n-1}\right]$ exactly $n$ times in unit time.

Since the $H_{n}$ 's are constant on each torus, we henceforth write $H_{n}[M]$ for their values on $M$.

\section{Stability Result}

The Camassa-Holm flow preserves the torus $M \subset C^{\infty}(\mathrm{S})$. Moreover, due to the integrability of (1.1), $M$ can be viewed as a Jacobi variety on which the flow is just straight line motion. Since the lines of motion in general are irrationally inclined to the directions represented by the primitive periods of the variety, the typical trajectory of (1.1) is dense in $M$. Therefore, the best kind of stability we can hope for in general is the following: If two functions $m$ and $\bar{m}$ are close, then so are the tori on which they lie. The next theorem states that the Camassa-Holm equation is stable in this sense.

Theorem 1. Let $0<m_{0} \in M, \epsilon>0$, and $k \geq 1$. There is a $\delta>0$ such that if $\bar{m}_{0} \in \bar{M}$ satisfies

$$
\left\|m_{0}-\bar{m}_{0}\right\|_{H^{k}(\boldsymbol{S})}<\delta,
$$

then $M$ and $\bar{M}$ are $\epsilon$-close in the $H^{k-2}$-norm, i.e. for any $\bar{m} \in \bar{M}$, there is an $m \in M$ such that

$$
\|m-\bar{m}\|_{H^{k-2}(\boldsymbol{S})}<\epsilon .
$$

In the case of a traveling wave solution our definition of stability implies the usual notion of orbital stability: Any solution starting close to a traveling wave remains close to a translate of it for all times. To see this observe that the spectrum associated to a traveling wave $\varphi(x-c t) \in M$ has only the first gap open, $\lambda_{2}<\lambda_{1}$, whereas all other eigenvalues except $\lambda_{0}$ are double (cf. [12]). Thus the torus $M$ can be identified with the circle of translates of $\varphi$. If $\bar{m} \in \bar{M}$ starts close to $m$, then the stability ensures that $M$ and $\bar{M}$ are close. Consequently, the trajectory of $\bar{m}$ always stays close to a translate of $m$.

For each speed $c>0$ equation (1.1) admits a two-parameter family of smooth periodic traveling wave solutions [24]. Since they all have positive 
momentum $m=u-u_{x x}>0$ (see [23]), their orbital stability follows from Theorem 1.

THEOREM 2. The smooth periodic traveling wave solutions of the CamassaHolm equation are orbitally stable.

\section{Proof of Stability}

In this section we prove Theorem 1. The proof employs the trace formula (see [6])

$$
u(x)=\frac{\cosh (1 / 2)}{2 \sinh (1 / 2)} H_{0}[M]+\sum_{n \geq 1} \frac{1}{\mu_{n}(x)},
$$

where the $\mu_{n}$ 's are as in Lemma 1: $\mu_{n}(x)$ is the $n$th Dirichlet eigenvalue associated to the potential $m(\cdot+x)$. First we need a lemma that deals with the tail of the sum in (7.1).

LEMma 2. We have

$$
u(x)=\frac{\cosh (1 / 2)}{2 \sinh (1 / 2)} H_{0}[M]+\sum_{n=1}^{N} \frac{1}{\mu_{n}(x)}+o(1),
$$

$$
u_{x}(x)=-\sum_{n=1}^{N} \frac{\mu_{n}^{\prime}(x)}{\mu_{n}^{2}(x)}+o(1), \quad \ldots, \quad u^{(k)}(x)=\sum_{n=1}^{N}\left(\frac{1}{\mu_{n}(x)}\right)^{(k)}+o(1),
$$

with o(1) approaching 0 uniformly in an $H^{k}$-neighborhood of $M$ as $N \uparrow \infty$.

Proof. The continuity of $H_{-1}$ in the $H^{k}$-norm and the asymptotic formula (4.5) immediately give (7.2).

To show the differentiated formulas (7.3) we need to study the $\mu_{n}$ 's in more detail. In view of (3.3) the differential equations (5.3) can be spelled out as

$$
\mu_{n}^{\prime}= \pm \mu_{n} \sqrt{\frac{\mu_{n}}{\lambda_{0}}-1} \sqrt{\left(1-\frac{\mu_{n}}{\lambda_{2 n-1}}\right)\left(\frac{\mu_{n}}{\lambda_{2 n}}-1\right)} \prod_{1 \leq m \neq n} \frac{\sqrt{\left(1-\frac{\mu_{n}}{\lambda_{2 m-1}}\right)\left(1-\frac{\mu_{n}}{\lambda_{2 m}}\right)}}{1-\frac{\mu_{n}}{\mu_{m}}} .
$$

We rewrite this as

$$
\begin{aligned}
& \text { (7.4) } \mu_{n}^{\prime}= \pm \frac{1}{\sqrt{-\lambda_{0}}} \prod_{m \geq 1} \frac{-\mu_{m}}{\sqrt{\lambda_{2 m-1} \lambda_{2 m}}} \\
& \times \sqrt{\lambda_{0}-\mu_{n}} \sqrt{\left(\lambda_{2 n-1}-\mu_{n}\right)\left(\mu_{n}-\lambda_{2 n}\right)} \prod_{1 \leq m \neq n} \frac{\sqrt{\left(\lambda_{2 m-1}-\mu_{n}\right)\left(\lambda_{2 m}-\mu_{n}\right)}}{\mu_{n}-\mu_{m}} .
\end{aligned}
$$


Using the trace formula [12]

$$
\sqrt{m(x)}=\frac{1}{2 \sqrt{-\lambda_{0}}} \prod_{m \geq 1} \frac{-\mu_{m}}{\sqrt{\lambda_{2 m-1} \lambda_{2 m}}},
$$

we obtain

$$
\begin{aligned}
\mu_{n}^{\prime}= & \pm 2 \sqrt{m(x)} \sqrt{\lambda_{0}-\mu_{n}} \\
& \times \sqrt{\left(\lambda_{2 n-1}-\mu_{n}\right)\left(\mu_{n}-\lambda_{2 n}\right)} \prod_{1 \leq m \neq n} \frac{\sqrt{\left(\lambda_{2 m-1}-\mu_{n}\right)\left(\lambda_{2 m}-\mu_{n}\right)}}{\mu_{n}-\mu_{m}} .
\end{aligned}
$$

Note that, in view of the continuity of $H_{-1}[m]$ and $H_{-2}[m]$ in the $H^{k}$-norm, (4.5) yields

$$
\begin{gathered}
\sqrt{\lambda_{0}-\mu_{n}}=O(n), \quad \sqrt{\left(\lambda_{2 n-1}-\mu_{n}\right)\left(\mu_{n}-\lambda_{2 n}\right)}=O(1), \\
\prod_{1 \leq m \neq n} \frac{\sqrt{\left(\lambda_{2 m-1}-\mu_{n}\right)\left(\lambda_{2 m}-\mu_{n}\right)}}{\mu_{n}-\mu_{m}}=O(1),
\end{gathered}
$$

as $n \uparrow \infty$ uniformly for $m$ in the vicinity of $M$. Hence $\mu_{n}^{\prime}=O(n)$ as $n \uparrow \infty$, so that the formula for $u_{x}$ follows by differentiation of (7.1).

Since all the functionals $H_{-1}[m], H_{-2}[m], \ldots, H_{-k-1}[m]$ are continuous in the $H^{k}$-norm (cf. [25]), we obtain the uniform estimates

$$
\sqrt{\lambda_{2 n-1}-\mu_{n}}=O\left(n^{-k+1}\right), \quad \sqrt{\mu_{n}-\lambda_{2 n}}=O\left(n^{-k+1}\right) .
$$

Differentiating (7.5) and employing these estimates, we arrive at the formulas for the higher derivatives of $u$.

Now suppose $m_{0} \in M$ and $\bar{m}_{0} \in \bar{M}$ are two functions close in the $H^{k}$-norm. Let $\bar{m}=\bar{u}-\bar{u}_{x x}$ be a fixed but arbitrary point on $\bar{M}$. We want to construct $m=u-u_{x x} \in M$ close to $\bar{m}$.

Let the periodic spectrum associated to $m$ respectively $\bar{m}$ be $\cdots<\lambda_{2} \leq$ $\lambda_{1}<\lambda_{0}<0$ respectively $\cdots<\bar{\lambda}_{2} \leq \bar{\lambda}_{1}<\bar{\lambda}_{0}<0$. A point on $M$ is given by a sequence $\left\{\mu_{n}, \sqrt{\Delta^{2}\left(\mu_{n}\right)-1}\right\}$ of numbers $\mu_{n} \in\left[\lambda_{2 n}, \lambda_{2 n-1}\right]$ and signatures of the radicals $\sqrt{\Delta^{2}\left(\mu_{n}\right)-1}$. By Lemma 2 we have

$$
\begin{aligned}
u(x)-\bar{u}(x)=\frac{\cosh (1 / 2)}{2 \sinh (1 / 2)}\left(H_{0}[M]\right. & \left.-H_{0}[\bar{M}]\right) \\
& +\sum_{n=1}^{N}\left(\frac{1}{\mu_{n}(x)}-\frac{1}{\bar{\mu}_{n}(x)}\right)+o(1),
\end{aligned}
$$


where the $\bar{\mu}_{n}$ 's are the Dirichlet eigenvalues associated to $\bar{m}$. First observe that $\left|H_{0}[M]-H_{0}[\bar{M}]\right|$ can be made arbitrarily small by choice of $\bar{m}_{0}$. To show that the sum in (7.6) is small, we estimate the disturbance in the motion of $\mu_{n}$ due to a small change in the potential $m$.

Viewing (3.1) as an eigenvalue problem with periodic or antiperiodic boundary conditions, we infer from the Minimax principle that each $\lambda_{n}$ can be expressed as a quotient

$$
-\min \left\{\max _{\psi \in \mathscr{S}} \frac{\int_{\mathrm{S}}\left(\psi_{x}^{2}+\frac{1}{4} \psi^{2}\right) d x}{\int_{S} m \psi^{2} d x}\right\},
$$

where the minimum is taken over all subspaces $\mathscr{S}$ of a certain dimension (cf. [1]). This representation shows that the periodic spectrum depends continuously on $m$. Hence $\lambda_{n}$ is close to $\bar{\lambda}_{n}$ for all $n$, so that we may choose the initial conditions $\mu_{n}(0)$ close to $\bar{\mu}_{n}(0)$.

As in the proof of Lemma 1 presented in [6] we let $\mathbf{X}$ be the Banach space of points $\mathbf{x}=\left(x_{1}, x_{2}, \ldots\right) \in \mathbf{R}^{\infty}$ endowed with the norm

$$
\|\mathbf{x}\|=\sum_{n=1}^{\infty} \frac{\left|x_{n}\right|}{n^{3}}<\infty .
$$

Introducing new variables $x_{n}(t)$ by

$$
\mu_{n}(t)=\lambda_{2 n-1}+\left(\lambda_{2 n}-\lambda_{2 n-1}\right) \sin ^{2}\left(x_{n}(t)\right),
$$

the equations (7.4) can be written as

$$
\frac{d x_{n}}{d t}=F_{n}\left(x_{1}, x_{2}, \ldots\right), \quad n \geq 1,
$$

where

$$
\begin{aligned}
F_{n}\left(x_{1}, x_{2}, \ldots\right)= \pm & \frac{1}{\sqrt{-\lambda_{0}}} \prod_{m \geq 1} \frac{-\mu_{m}}{\sqrt{\lambda_{2 m-1} \lambda_{2 m}}} \\
& \times \sqrt{\lambda_{0}-\mu_{n}} \prod_{1 \leq m \neq n} \frac{\sqrt{\left(\lambda_{2 m-1}-\mu_{n}\right)\left(\lambda_{2 m}-\mu_{n}\right)}}{\mu_{n}-\mu_{m}}
\end{aligned}
$$

Similarly, the motion of $\bar{\mu}_{n}(x)=\bar{\lambda}_{2 n-1}+\left(\bar{\lambda}_{2 n}-\bar{\lambda}_{2 n-1}\right) \sin ^{2}\left(\bar{x}_{n}(t)\right)$ is described by

$$
\frac{d \bar{x}_{n}}{d t}=\bar{F}_{n}\left(\bar{x}_{1}, \bar{x}_{2}, \ldots\right), \quad n \geq 1,
$$


where $\bar{F}_{n}$ is given by the same formula as $F_{n}$ except that the $\lambda$ 's are replaced by $\bar{\lambda}$ 's.

In [6] it was proved that $\mathbf{F}=\left(F_{1}, F_{2}, \ldots\right)$ satisfies the Lipschitz condition

$$
\|\mathbf{F}(\mathbf{x})-\mathbf{F}(\mathbf{y})\| \leq K\|\mathbf{x}-\mathbf{y}\|, \quad \mathbf{x}, \mathbf{y} \in \mathbf{X},
$$

for some constant $K>0$. Furthermore, from (7.7) we see that there is a $\rho>0$ such that

$$
\|\mathbf{F}(\mathbf{x})-\overline{\mathbf{F}}(\mathbf{x})\| \leq \rho, \quad \mathbf{x} \in \mathbf{X} .
$$

Hence standard ODE estimates show that

$$
\|\mathbf{x}(t)-\overline{\mathbf{x}}(t)\| \leq\|\mathbf{x}(0)-\overline{\mathbf{x}}(0)\| e^{K t}+\frac{\rho}{K}\left(e^{K t}-1\right), \quad t \geq 0 .
$$

As the $\bar{\lambda}$ 's approach the $\lambda$ 's, $\rho$ can be taken smaller and smaller. In particular, choosing $\left\|m_{0}-\bar{m}_{0}\right\|_{H^{k}(S)}$ small, $\mu_{n}(x)$ can be arranged to be arbitrarily close to $\bar{\mu}_{n}(x)$ for $0 \leq x<1$. Thus (7.6) shows that $\max _{x \in \mathrm{S}}|u(x)-\bar{u}(x)|$ is small. A similar argument can now be carried out for the derivatives of $\mu_{n}$, showing that $\max _{x \in \mathrm{S}}\left|\mu_{n}^{(j)}(x)-\bar{\mu}_{n}^{(j)}(x)\right|$ is small for $j \leq k$. The differentiated versions of (7.6) obtained from Lemma 2 therefore show that $\|u-\bar{u}\|_{C^{k}(S)}$ is small. But $C^{k}(\mathrm{~S}) \subset H^{k}(\mathrm{~S})$ so we deduce that

$$
\|m-\bar{m}\|_{H^{k-2}(\mathbf{S})}=\|u-\bar{u}\|_{H^{k}(\mathbf{S})}
$$

is small. This finishes the proof.

\section{REFERENCES}

1. Blanchard, P., and Brüning, E., Variational Methods in Mathematical Physics, SpringerVerlag, Berlin, 1992.

2. Camassa, R., and Holm, D., An integrable shallow water equation with peaked solitons, Phys. Rev. Lett. 71 (1993), 1661-1664.

3. Camassa, R., Holm, D., and Hyman, J., A new integrable shallow water equation, Adv. Appl. Mech. 31 (1994), 1-33.

4. Constantin, A., A general-weighted Sturm-Liouville problem, Ann. Scuola Norm. Sup. Pisa Cl. Sci. (4) 24 (1997), 767-782.

5. Constantin, A., On the Cauchy problem for the periodic Camassa-Holm equation, J. Differential Equations 141 (1997), 218-235.

6. Constantin, A., On the inverse spectral problem for the Camassa-Holm equation, J. Funct. Anal. 155 (1998), 352-363.

7. Constantin, A., and Escher, J., Wave breaking for nonlinear nonlocal shallow water equations, Acta Math. 181 (1998), 229-243.

8. Constantin, A., and Escher, J., Well-posedness, global existence, and blowup phenomena for a periodic quasi-linear hyperbolic equation, Comm. Pure Appl. Math 51 (1998), 475-504. 
9. Constantin, A., and Escher, J., On the blow-up rate and the blow-up set of breaking waves for a shallow water equation, Math. Z. 233 (2000), 75-91.

10. Constantin, A., and Kolev, B., On the geometric approach to the motion of inertial mechanical systems, J. Phys. A 35 (2002), R51-R79.

11. Constantin, A., and Kolev, B., Geodesic flow on the diffeomorphism group of the circle, Comment. Math. Helv. 78 (2003), 787-804.

12. Constantin, A., and McKean, H., A shallow water equation on the circle, Comm. Pure Appl. Math. 52 (1999), 949-982.

13. Constantin, A., and Molinet, L., Global weak solutions for a shallow water equation, Comm. Math. Phys. 211 (2000), 45-61.

14. Constantin, A., and Molinet, L., Orbital stability of solitary waves for a shallow water equation, Phys. D 157 (2001), 75-89.

15. Constantin, A., and Strauss, W., Stability of peakons, Comm. Pure Appl. Math. 53 (2000), 603-610.

16. Constantin, A., and Strauss, W., Stability of the Camassa-Holm solitons, J. Nonlinear Sci. 12 (2002), 415-422.

17. Dai, H., Model equations for nonlinear dispersive waves in a compressible Mooney-Rivlin rod, Acta Mech. 127 (1998), 193-207.

18. Danchin, R., A few remarks on the Camassa-Holm equation, Differential Integral Equations 14 (2001), 953-988.

19. Fuchssteiner, B., and Fokas, A., Symplectic structures, their Bäcklund transformation and hereditary symmetries, Phys. D 4 (1981), 47-66.

20. Gesztesy, F., and Holden, H., Algebro-geometric solutions of the Camassa-Holm hierarchy, Rev. Mat. Iberoamericana 19 (2003), 73-142.

21. Lenells, J., Stability of periodic peakons, Internat. Math. Res. Notices 10 (2004), 485-499.

22. Lenells, J., A variational approach to the stability of periodic peakons, J. Nonlinear Math. Phys. 11 (2004), 151-163.

23. Lenells, J., Traveling wave solutions of the Camassa-Holm and Korteweg-de Vries equations, J. Nonlinear Math. Phys. 11 (2004), 508-520.

24. Lenells, J., Traveling wave solutions of the Camassa-Holm equation, J. Differential Equations, to appear.

25. Lenells, J., Conservation laws of the Camassa-Holm equation, J. Phys. A 38 (2005), 869-880.

26. Lenells, J., The Correspondence between KdV and Camassa-Holm, Internat. Math. Res. Notices, 71 (2004), 3797-3811.

27. Li, Y., and Olver, P., Well-posedness and blow-up solutions for an integrable nonlinearly dispersive model wave equation, J. Differential Equations 162 (2000), 27-63.

28. Magnus, W., and Winkler, S., Hill's Equation, John Wiley \& Sons, 1966.

29. McKean, H., Stability for the Korteweg-De Vries Equation, Comm. Pure Appl. Math. 30 (1977), 347-353.

30. McKean, H., Breakdown of a shallow water equation, Asian J. Math. 2 (1998), 867-874.

31. McKean, H., and van Moerbeke, P., The spectrum of Hill's equation, Invent. Math. 30 (1975), 217-274.

32. Misiolek, G., A shallow water equation as a geodesic flow on the Bott-Virasoro group, J. Geom. Phys. 24 (1998), 203-208.

33. Rodriguez-Blanco, G., On the Cauchy problem for the Camassa-Holm equation, Nonlinear Anal. 46 (2001), 309-327. 
34. Trubowitz, E., The Inverse Problem for Periodic Potentials, Comm. Pure Appl. Math. 30 (1977), 321-337.

\author{
DEPARTMENT OF MATHEMATICS \\ LUND UNIVERSITY \\ PO BOX 118 \\ 22100 LUND \\ SWEDEN \\ E-mail: jonatan@maths.lth.se
}

